\title{
CARACTERIZACIÓN DE LOS MATERIALES SEDIMENTARIOS INCONSOLIDADOS COSTEROS Y ALUVIALES ACTUALES EN LA REGIÓN DE PARRITA, PACÍFICO CENTRAL DE COSTA RICA
}

\author{
Guaria Cárdenes Sandí
Escuela Centroamericana de Geología, Apdo. 214-2060 Universidad de Costa Rica gcardene@geologia.ucr.ac.cr

(Recibido 29/4/03; aceptado 8/8/03)

\begin{abstract}
Two different sedimentary environments (alluvial and coastal setting) have been recognized in the Parrita area. The continental environment is represented by alluvial facies (alluvial plains, active meandric channels, active braided river channels, and paleo-meandric channel facies), and small lacustrine deposits. The coastal environment is conformed by beach, sand bars, and estuarine facies. The beach facies are formed by the assign of the littoral currents (NW-SE), which carry out the sediment from the adjacent coast and may be from the inner platform. The sand bars and estuarine facies constitute a bar barrier system. This system is formed by a sequence of sand bars and swamps, which are colonized by "mangle" vegetation. The granulometric characteristics of sediments (mean, mode, sorting, skewness, kurtosis) typify every sedimentary facies.
\end{abstract}

Keywords: Alluvial plains, coastal setting, granulometric characteristics, lacustrine deposits, estuary, Parrita, Costa Rica, meandric channels, braided channels.

RESUMEN: Se reconocen dos tipos de ambientes sedimentarios, los continentales y los costeros o de transición. Los primeros tienen una predominancia espacial sobre los ambientes de transición. Los ambientes continentales están representados principalmente por depósitos aluviales (facies de planicie aluvial, de canal activo tipo meándrico, de canal activo tipo braided river, y de paleo-canal meándrico) y en mucha menor proporción por sedimentación lacustre (facies lacustres). Las facies que se desarrollan dentro del ambiente costero de la zona son facies de playa, de barra arenosa y estuarinas. Las facies de playa se depositan por la acción de las corrientes litorales que tienen una dirección de NW a SE. Las facies de barra arenosa y las estuarinas constituyen sistemas de islas barreras. Estos sistemas se caracterizan por la presencia de una serie de barras arenosas tras las cuales se instala una laguna costera (lagoon), que ha sido colonizado por vegetación tipo "mangle". Los sedimentos, tanto continentales como costeros, presentan características granulométricas (moda, mediana, selección, asimetría y distribución poblacional) homogéneas dentro de cada una de las diferentes facies sedimentarias.

Palabras clave: Planos aluviales, ambientes costeros, características granulométricas, depósitos lacustres, estuarios, Parrita, Costa Rica, canales meándricos, canales trenzados. 


\section{INTRODUCCIÓN}

Se realiza una caracterización granulométrica y en parte sedimentológica de los materiales sedimentarios inconsolidados en la región de Parrita, en el pacífico central costarricense (Fig. 1). Se tipificaron los materiales por medio de análisis granulométricos (aprox. 80 muestras), análisis de fotografías aéreas y trabajo de campo. Con esta información se elaboró un mapa de facies sedimentarias superficiales; además, se estableció una serie de características (granulométricas, de distribución espacial, etc.) que distinguen las diferentes facies definidas.

Las facies sedimentarias que se encuentran en la zona de estudio corresponden con ambientes tanto continentales como marinos de transición. La distribución de facies mostrada en la figura 1 corresponde con las facies en superficie y no toma en cuenta los cambios de facies en profundidad. Es importante aclarar que en este mapa las rocas duras corresponden con las formaciones geológicas, constituidas por material consolidado, que ha experimentado en mayor o menor grado los procesos diagenéticos, ígneos o metamórficos.

\section{AMBIENTES CONTINENTALES}

Los ambientes sedimentarios continentales en la zona de estudio tienen una fuerte prevalencia espacial sobre los ambientes de transición. Están representados principalmente por depósitos aluviales y en mucha menor proporción por sedimentación lacustre.

En la planicie aluvial de Parrita y sus alrededores los cursos de agua con carga mixta tienen patrones trenzados con canales poco sinuosos, pero en su mayoría se desarrollan canales tipo

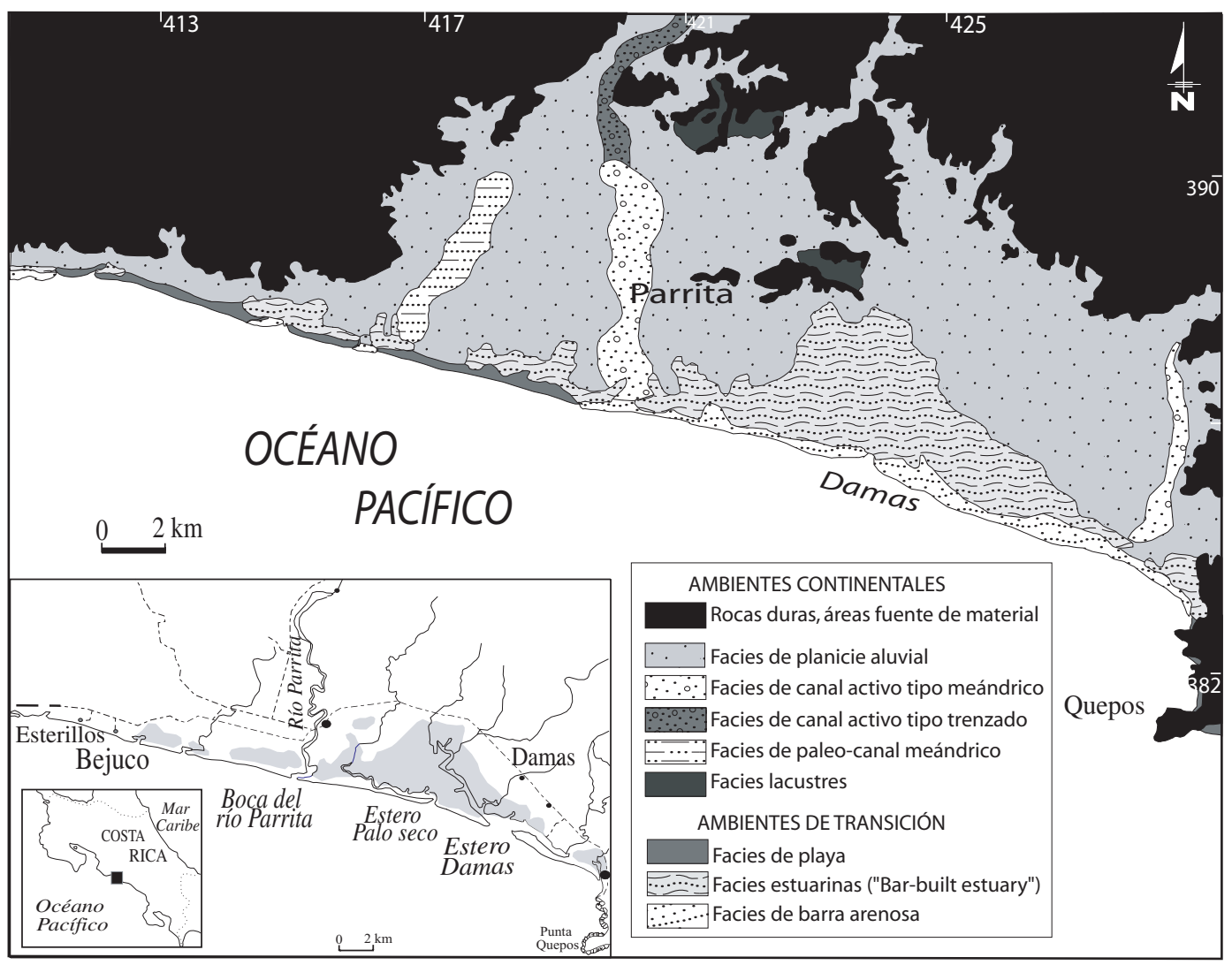

Fig. 1: Mapa de facies sedimentarias actuales. 
meándrico. Los canales meándricos se presentan en fajas o cinturones discretos a lo largo de la planicie aluvial. Los ríos de carga mixta transportan sedimentos finos en suspensión, así como una carga de fondo. Su carga de fondo es principalmente arenosa con una pequeña fracción de gravas $(<10 \%)$.

\section{Facies de planicie aluvial}

La sedimentación de planos aluviales en Costa Rica está poco documentada. Muchas áreas están intensivamente utilizadas y esto produce limitantes al hacer estudios, ya sea por la mala exposición de los depósitos o, porque han sido removidos para ser cultivados o por el pastoreo. Las facies de planicie aluvial son las más ampliamente distribuidas en la región (Fig. 1). En general los sedimentos presentan coloraciones café oscuro o claro.

Esta constituida por materiales sedimentarios con fracciones gruesas de hasta $-5,25 \varnothing$ (ver apéndice). Sin embargo, como se puede ver en las figuras 2 y 3 los sedimentos que conforman la planicie aluvial son en su mayoría materiales finos con diámetros menores a los 3,75 Ø.
Prácticamente todos las muestras colectadas a lo largo de estas facies contienen fracciones finas (limos, arcillas) constituyendo más del 50\% y en algunos casos llegan a ser el $90 \%$ del sedimento.

Existen dos grupos principales de tipos granulométricos de sedimentos. En el grupo 1 las fracciones finas constituyen casi en su totalidad el sedimento $(>75 \%)$, siendo las fracciones más gruesas arenas gruesas o gravas marginales (Fig. 2). Se presentan sedimentos unimodales, con una tendencia asimétrica negativa, lo que indica niveles energéticos bajos (disminución en la capacidad de transporte). El principal mecanismo de transporte de estos sedimentos es la suspensión y en mucho menor grado la tracción (saltación principalmente).

En el grupo 2 (Fig. 3) la fracción de finos disminuye a menos de $50 \%$ y las fracciones gruesas son mucho más importantes, pudiendo alcanzar más del 50\%. La diferencia entre ambos puede partir del hecho que los sedimentos más finos del grupo 1 son depositados por desbordamiento de canales en un flujo laminar de baja energía, alejados estos de las áreas de canal; mientras que los sedimentos más gruesos de grupo 2 pueden ser depósitos producidos en la zona donde se inicia el desbordamiento, o corresponden con zonas
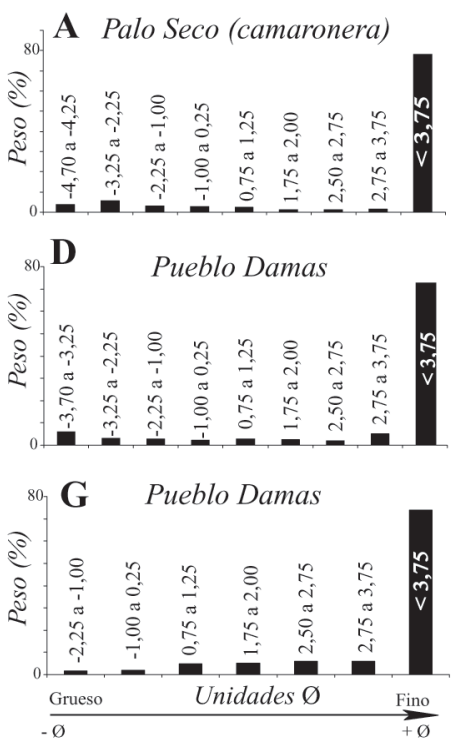
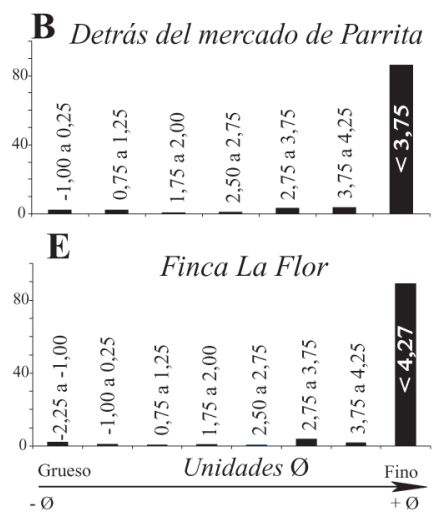

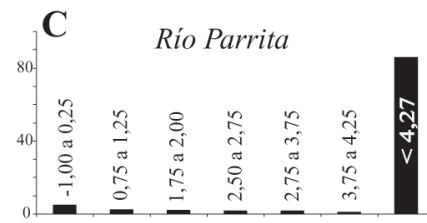

F

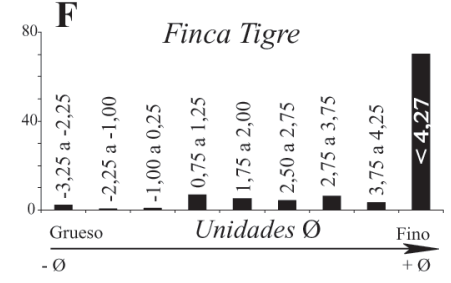

Fig. 2: Histograma de distribución granulométrica para las facies de planicie aluvial (grupo 1). 

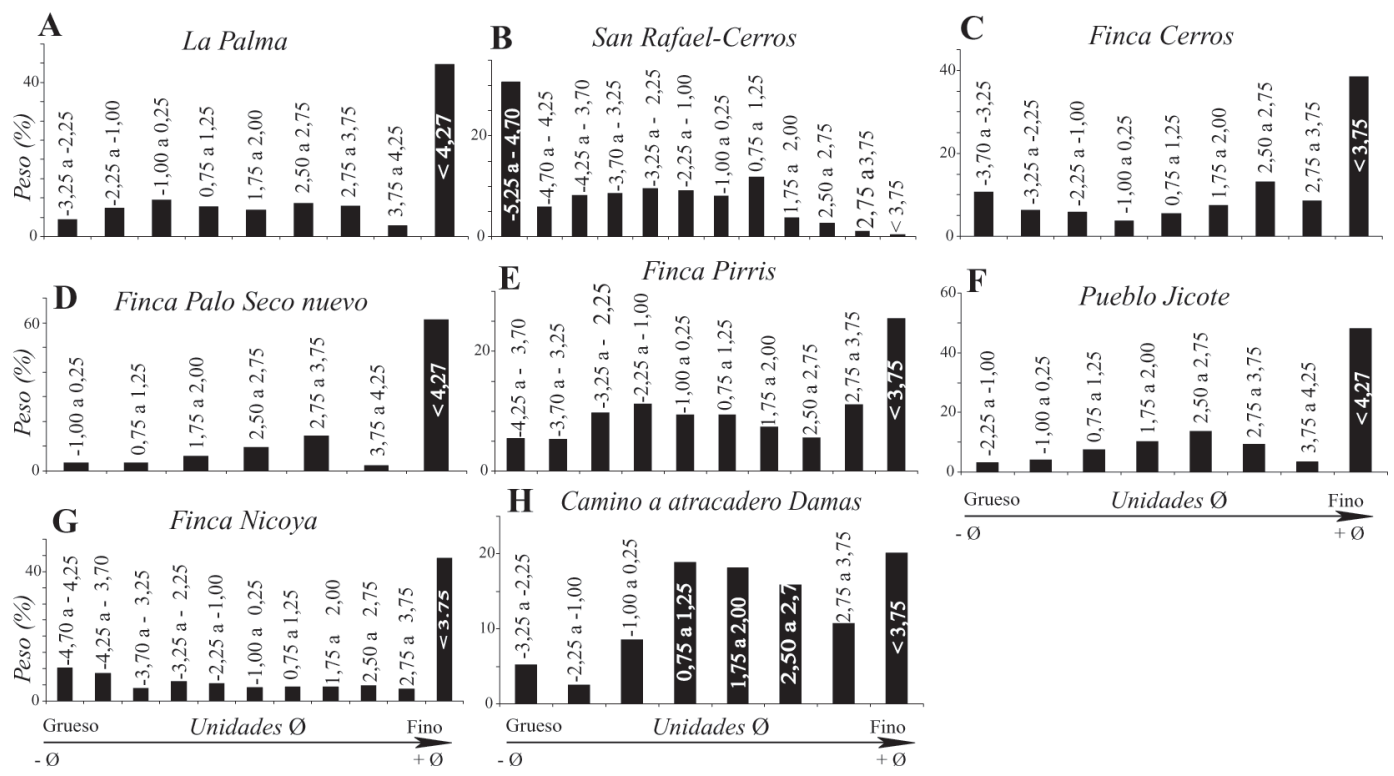

Fig. 3: Histograma de distribución granulométrica para las facies de planicie aluvial (grupo 2).

de paleo-canales de menor tamaño. En este grupo se presenta una distribución polimodal (Fig. 3), con asimetría negativa, no obstante siempre hay un predominio de la población de materiales finos $(<3,75 \varnothing)$. El rango de tamaños de grano aumenta considerablemente, desde $-5,25$ a $<4,27$ $\varnothing$. Esto indica una mayor energía en el medio, en comparación con el grupo 1. Aquí la suspensión sigue siendo un mecanismo importante de transporte, sin embargo, hay un aumento de la tracción (saltación y rodamiento).

La moda corresponde con los tamaños menores 3,75 a 4,27 Ø, la selección es buena, tiene valores que en su gran mayoría superan 1 (para las fórmulas utilizadas ver apéndice). Los valores de angulosidad varían considerablemente, sin embargo, se puede decir que son poco angulosas, lo cual indica un rango amplio de diámetros de partículas.

En cuanto a la génesis, los procesos sedimentarios fuera de la faja de meandros, o sea en la planicie aluvial están dominados por la acreción vertical derivada de un flujo de sedimento introducido a la planicie por canales o desbordamiento de los canales principales (depósitos de inundación), y en algunos casos el flujo de agua puede escapar de los canales secundarios (depósitos de crevasses splays). Es importante acotar que, a pesar de que la planicie aluvial es una zona donde principalmente se produce depositación, también, se establece un flujo en los planos aluviales que produce erosión y retrabajo de los últimos sedimentos depositados. Los procesos de depositación/erosión se desarrollan principalmente durante la estación lluviosa, época en la cual se producen fuertes eventos de inundación en la zona.

\section{Facies de canal activo tipo meándrico}

Estos depósitos corresponden con los actuales canales de los principales ríos, los cuales presentan un patrón de drenaje meándrico. Tienen una distribución espacial algo reducida, sin embargo, estas son las zonas más activas dentro de la planicie aluvial (Fig. 1). Los sedimentos tienen color café claro o beige.

Los materiales que pertenecen al río Parrita presentan escaso porcentaje de sedimento fino $(<3,75 \varnothing$ menos del $5 \%)$, mientras que tienen fracciones gruesas de hasta $-5,70 \emptyset$ que corresponden 
con más de 50\% (Fig. 4). Los ríos Cañas y Paquita presentan una mayor cantidad de finos en su matriz $(<3,75 \varnothing$ mayor al $70 \%)$. Los clastos corresponden con rocas ígneas, lavas en su mayoría y algunas areniscas y lutitas.

Los materiales de las facies de canal activo tipo meándrico se caracterizan por ser polimodales, con asimetrías tanto positivas como negativas, lo cual refleja una gran variabilidad del sedimento (Fig. 4). Esto nos sugiere que la energía dentro de estas facies es muy variable. En algunos casos (E y F, Fig. 4) la energía es baja, con la suspensión como mecanismo de transporte predominante. En el resto de las muestras (A a D y G, Fig. 4), la energía en el medio aumenta mucho y los principales mecanismos de transporte son por saltación y rodamiento.

La tendencia central (mediana y moda) varían considerablemente, lo cual refuerza lo dicho anteriormente de la gran diferencia textural de los sedimentos. La selección es de regular a mala, ya que presenta valores superiores a 1 . Tanto la asimetría como la angulosidad son muy variables, lo que también indica cambios fuertes en la textura del sedimento y por ende en la energía del medio sedimentario.
Las principales estructuras sedimentarias presentes en esta facies son gradaciones laterales inversas en los depósitos de point-bar, imbricación de clastos, laminaciones paralelas y cruzadas. Los canales meándricos sinuosos están dominados por los procesos de acreción lateral, proceso por medio del cual se forman depósitos de point-bar principalmente.

\section{Facies de paleo-canal meándrico}

Con una distribución espacial limitada se localizan en la parte Oeste de la planicie aluvial (Fig. 1). En la figura 4 gráfica $\mathrm{H}$ se observa la muestra correspondiente a esta facies, la cual fue tomada en un antiguo meandro el cual ha sido utilizado parcialmente por la actual red de drenaje. Está formada por sedimentos con distribuciones polimodales, con tamaños de grano entre $-4,70$ y $<2,00 \varnothing$. Es posible reconocer al menos tres poblaciones principales, a saber: de $-4,70$ a $-3,25$, de $-3,25$ a 0,5 y la última de $0,25 \mathrm{a}<2,00 \varnothing$ (H, Fig. 4). El principal mecanismos de transporte es la tracción, el sedimento movilizado por suspensión es escaso.

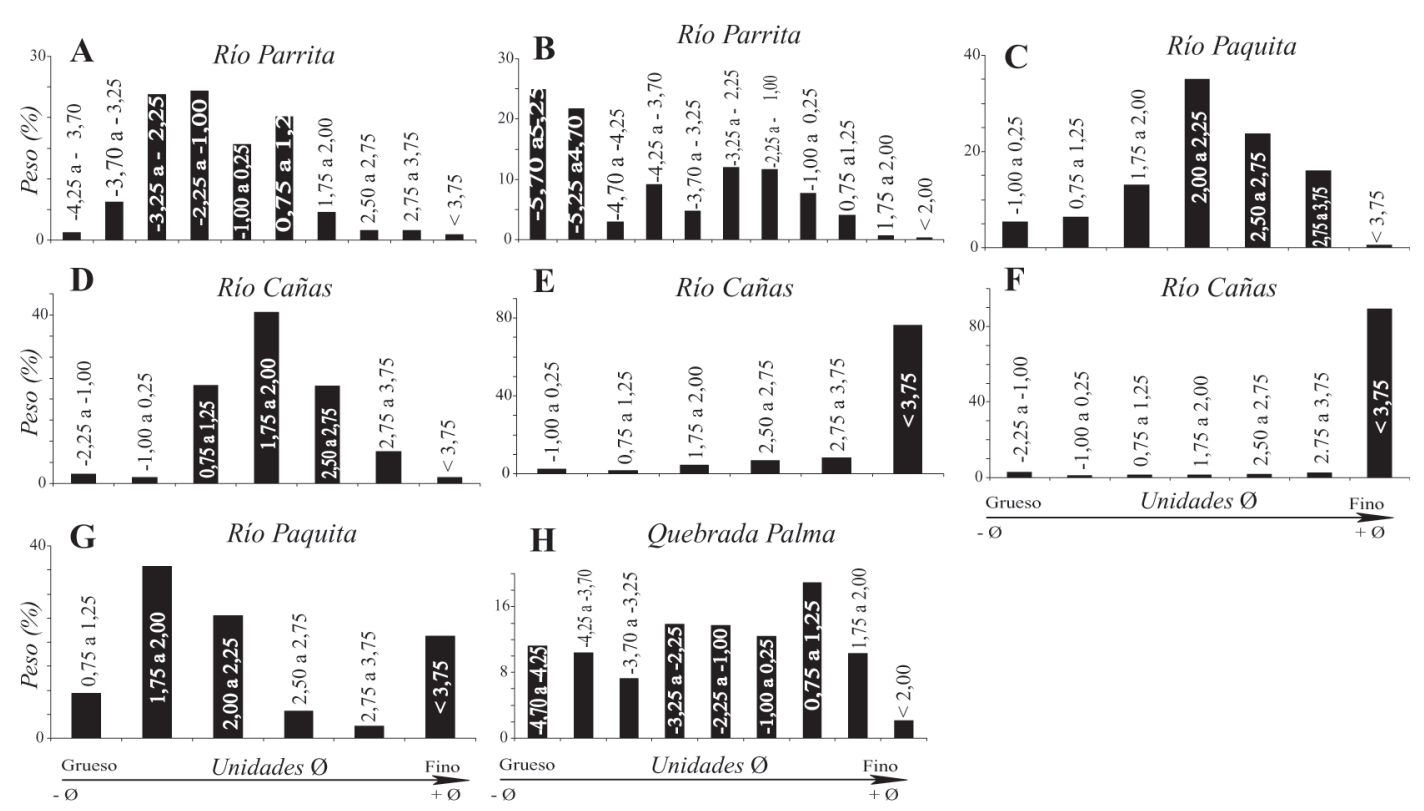

Fig. 4: Histograma de distribución granulométrica para las facies de canal activo tipo meándrico (A-G) y paleo-canal meándrico (H). 
En cuanto a los parámetros de tendencia central, la mediana es de 3,00 y la moda tiene un rango entre 0,75 y 1,25 . La curva es angulosa y la asimetría en las diferentes poblaciones es marcadamente positiva.

Las fracciones granulométricas van de los $-4,70$ a $<2,00 \varnothing$, siendo las más importantes las arenas medias a finas $(<1,00 \emptyset)$. En las zonas utilizadas por la red hídrica actual, los sedimentos que se depositan son de fondo de canal, gruesos (arenas y gravas). En las zonas colonizadas por vegetación se producen pequeñas zonas de pantano con la forma de media luna dejadas por los meandros abandonados.

No es posible observar estructuras sedimentarias debido a la colonización de la vegetación, a los cultivos instalados en la zona o a la incorporación de algunos tramos de la antigua faja de meandros como parte de la actual red hídrica, esto en forma de pequeños cauces.

Los canales meándricos sinuosos están dominados por los procesos de acreción lateral y migración de canales con procesos de abandono y cambio de curso del canal principal. Estas facies se generan cuando no solo se produjo la migración del cauce dentro de la faja de meandros, sino también dentro de la llanura aluvial, por un proceso de migración lateral. Actualmente, estas facies están siendo modificadas y en parte erosionadas por los procesos sedimentarios naturales y por el uso del suelo en la zona (cultivos).

\section{Facies de canal activo tipo "Braided river"}

Tienen una distribución bastante restringida y se localizan exclusivamente en la parte alta de río Parrita (Fig. 1). Presentan una amplia distribución granulométrica desde bloques hasta limos y arcillas en menor proporción. La gráfica de la figura 5 representa la distribución granulométrica de la matriz. La matriz es gruesa, tiene tamaños de grano que oscilan entre $-5,25$ y < $3,75 \varnothing$. Los clastos de mayor tamaño alcanzan dimensiones de $60 \times 80 \mathrm{~cm}$, pero la moda estadística corresponde con clastos de menos de 30 $\mathrm{cm}$. En general, la composición de los mismos es de rocas intrusivas ácidas, lavas andesíticas, con algunas areniscas y lutitas.

Las muestras colectadas en estas facies son polimodales, con asimetrías positivas (A, B, E y F, Fig. 5) y negativas (C y D, Fig. 5). Los mecanismos de transporte son tanto la suspensión como la tracción, siendo este último el más importante en la mayoría de las muestras.

Los parámetros de tendencia central son muy variables. La moda tiene valores que generalmente son superiores a $1,00 \varnothing$, lo que corresponde con arenas y gravas. La selección es muy mala, con valores entre 1,4 y 3,5. La asimetría y la angulosidad tienen valores que cambian mucho de una muestra a otra, pero son curvas angulosas (A a D, Fig. 5) y curvas más bien achatadas (E y F, Fig. 5). Los amplios rangos
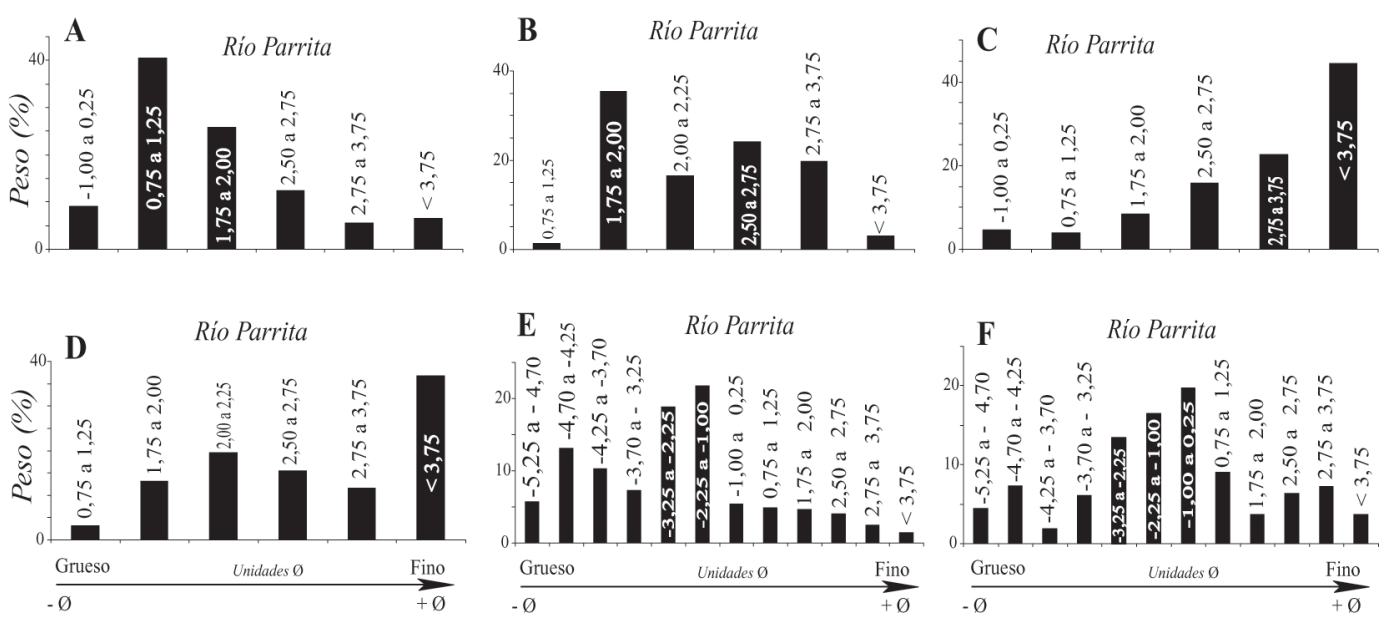

Fig. 5: Histograma de distribución granulométrica para las facies de canal activo tipo braided river. 
de los parámetros en estas facies son un reflejo de la gran heterogeneidad de los materiales que se depositan, debido a los cambios en la energía que transporta los sedimentos a largo y ancho del cauce.

Los canales trenzados con baja sinuosidad que se presentan en esta parte del río Parrita están dominados por acreción lateral, vertical y longitudinal, la cual conlleva la formación de barras laterales y longitudinales, como principales estructuras depositacionales.

Los estructuras sedimentarias más frecuentes son la imbricación de clastos y gradación inversa lateral en las barras longitudinales y laterales.

\section{Facies lacustres}

Esta facies está restringida al Este de la parte alta de río Parrita (Fig. 1) y a una zona entre un pequeño sistema de lomas al Este del poblado de Parrita (Las Vueltas). Se presenta como pequeñas lagunas o laguitos.

Tienen extensiones que no sobrepasan $500 \mathrm{~m}$ de diámetro, pero por lo general solo ocupan algunos cuantos metros, con profundidades que casi nunca sobrepasan el metro. Con pocas excepciones constituyen lagunas intermitentes, las cuales en el verano solo se presentan como zonas con humedad y mucha vegetación, mientras que en la estación lluviosa forman verdaderas lagunas.

En cuanto a las características granulométricas (Fig. 6), son sedimentos compuestos en su gran mayoría ( $>75 \%)$ de arenas muy finas y arcillas $(<3,75 \varnothing)$, lo cual es normal en sedimentos lacustres. Las fracciones más gruesas $(-2,25 \mathrm{a}$
$3,75 \varnothing)$ constituyen menos del $30 \%$ del sedimento. Los materiales $<3,75 \varnothing$ constituyen el $70 \%$, lo que implica una buena selección.

Los sedimentos lacustres tienen características muy homogéneas. Las curvas tienen asimetría negativa bien marcada (Fig. 6), lo que indica que el mecanismo de transporte dominante es la suspensión, lo cual coincide con los procesos de agradación que se producen durante la sedimentación en lacustres como los analizados.

También existe una fuerte coincidencia entre los parámetros de tendencia central, la mediana oscila entre 0,052 y 0,070 , la moda se encuentra en el intervalo de $<3,75 \varnothing$. La selección o sorteo es muy buena, con valores que van de 1,09 a 1,85. Las curvas son angulosas, lo que implica un rango de tamaños limitado.

El material tiene el característico color gris de sedimentos con alto contenido de materia orgánica, además, de la presencia de ramitas y hojas entre las capas de sedimento. En estos lagos y lagunillas se da la depositación de sedimento y materia orgánica y por la compactación de capas de sedimentos finos o sedimentos ricos en materia orgánica se tienden a colmatar.

Las estructuras sedimentarias presentes son sobre todo laminaciones paralelas y estratificaciones centimétricas, con algunas gradaciones normales.

En cuanto a la formación de estas facies, en zonas con climas húmedos y con precipitación considerable como las del área de Parrita, donde el flujo del río está cerca de su nivel base, algunos de los sedimentos de planos aluviales permanecen húmedos con lagos o suampos, siendo estos elementos importantes en la morfología de zona. Donde la vegetación es abundante
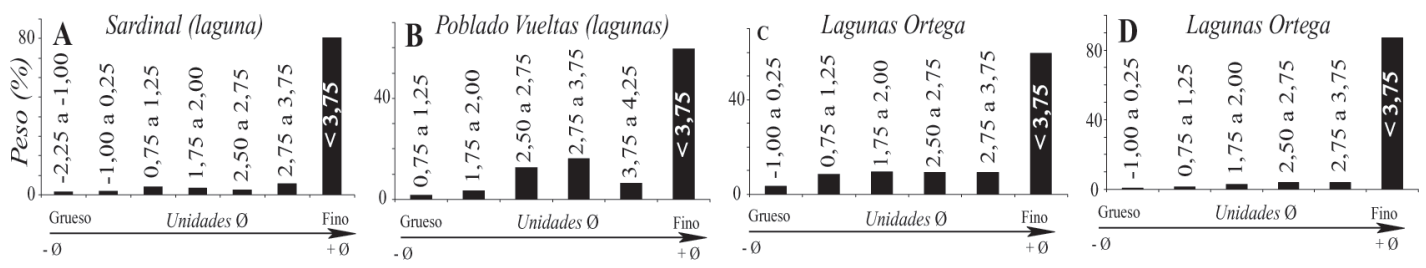

Fig. 6: Histograma de distribución granulométrica para las facies lacustres. 
y el nivel freático esta alto, la materia orgánica se acumula, especialmente si el sedimento en suspensión es detenido por las plantas que se encuentran en los márgenes del suampo. Los planos aluviales son inundados ya sea por el rebalse de los ríos o por el levantamiento del nivel freático lo cual conlleva la formación de lagos de planos aluviales tal y como los que se presentan en la planicie aluvial de Parrita de forma aislada. Estos pueden resultar de la intensa precipitación en los planos aluviales, que caracteriza la época lluviosa de la zona.

\section{AMBIENTES COSTEROS O DE TRANSICIÓN}

La zona costera es el área de transición donde el mar se junta con la tierra; es la región que está directamente influenciada por los procesos marinos. La zona costera se extiende hasta el quiebre de la plataforma continental y tierra adentro en el primer cambio topográfico fuerte sobre las mayores olas de tormenta, esta zona incluye la desembocadura de los ríos.

\section{Facies de playa}

Son café claro a beige. Tienen una buena distribución granulométrica, con fracciones que van desde los 1,75 Ø hasta las más finas menores a 3,75 Ø (Fig. 7); sin embargo, la fracción predominante es 2,50 a 2,75 Ø (arenas medias).

Todas las curvas granulométricas tienen comportamientos semejantes (A a D y F, Fig. 7). Están formadas principalmente por arenas finas depositadas por el accionar de las olas y las mareas; el sedimento es transportado principalmente por las corrientes litorales. En la costa se produce el transporte tanto por suspensión como por tracción.

La moda corresponde con los tamaños entre 2,50 y $2,75 \varnothing$, la selección tiene valores que superan $1(1,2$ a 1,6$)$, lo que indica que es regular. En cuanto a la angulosidad y asimetría las curvas son achatadas y simétricas, excepto $\mathrm{C}$ que tiene asimetría positiva y E con tendencia asimétrica negativa (Fig. 7).

Las principales estructuras sedimentarias que se presentan son riples, laminación paralela y cruzada y crecent marks; estas últimas son
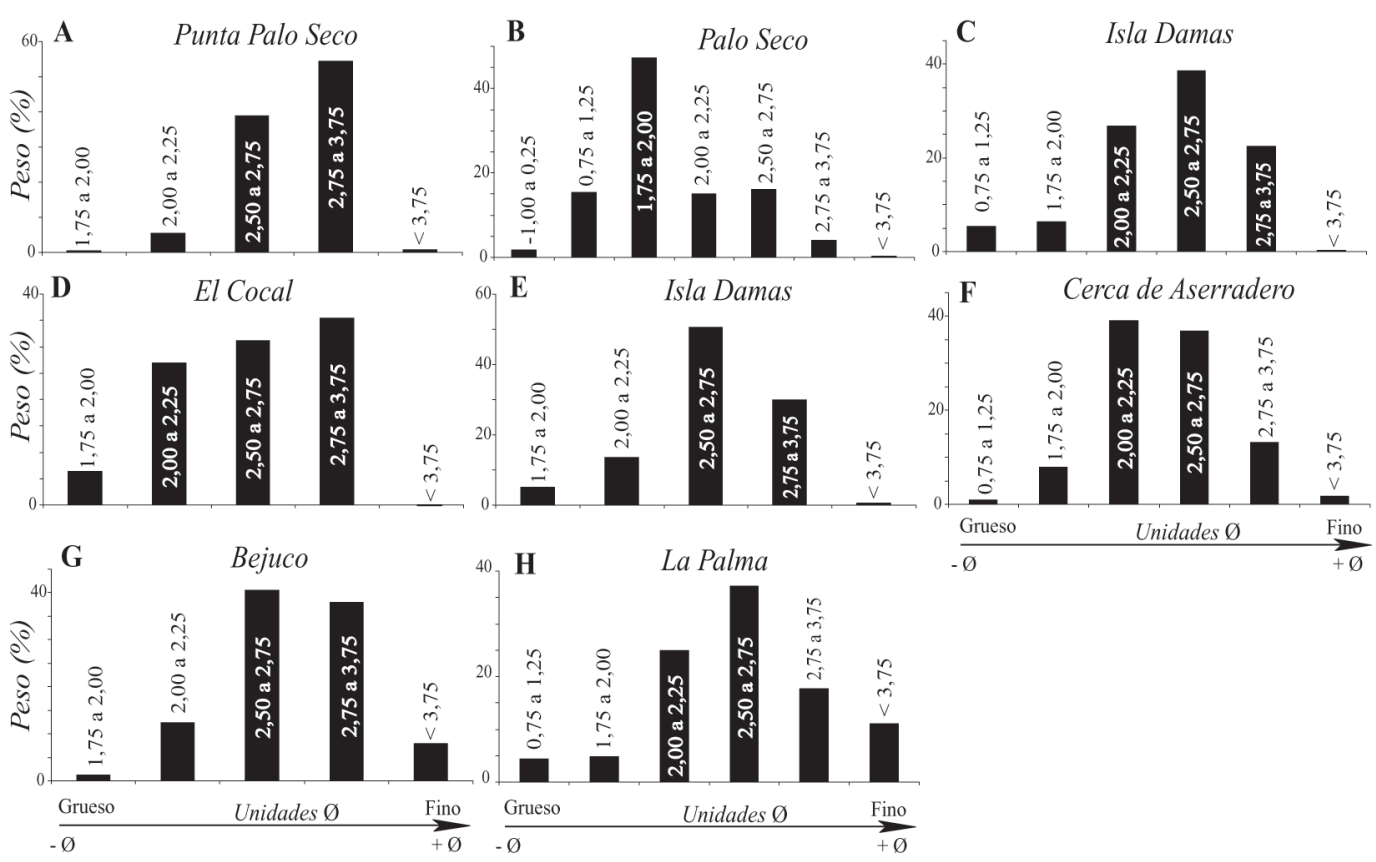

Fig. 7: Histograma de distribución granulométrica para las facies de playa. 
producidas por clastos centimétricos de rocas sedimentarias e ígneas ácidas y por conchas de organismos marinos (bivalvos). Los planos intermareales oscilan entre 80 y $25 \mathrm{~m}$. La berma permanente puede llegar a tener 20 a $30 \mathrm{~cm}$ de altura o estar ausente en algunos tramos, en los casos que se presenta se encuentra colonizada por pastos de muy baja densidad.

Las corrientes litorales tienen una dirección de NW a SE, por lo que una importante fuente de material para la playa y la anteplaya es el transporte a lo largo de la orilla en las zonas de oleaje y en la zona intermareal. El material retrabajado y el movimiento a través de la plataforma interna (hacia la playa) son mecanismos que también contribuyen a la construcción de estas facies de playa.

\section{Facies estuarinas (bar-built estuary)}

Las desembocaduras de los ríos en la zona de estudio corresponden con sistemas estuarinos, en los cuales se desarrolla un ecosistema de manglar. Pueden ser clasificados como bar-built estuary debido a que se da la presencia de barras arenosas que dan la protección necesaria para que se desarrollen los estuarios y sean colonizados por flora y fauna. Tienen una buena distribución a lo largo de toda la costa (Fig. 1), siendo el más importante el estero Damas-Palo Seco.

Los sedimentos estuarinos se pueden clasificar en dos grupos (Figs. 8 y 9). El grupo 1 está formado por los sedimentos que tienen más de un $70 \%$ de finos $(<3,75 \varnothing)$, como el caso de los gráficos $\mathrm{A}, \mathrm{B}, \mathrm{C}$ y $\mathrm{D}$ en la figura 8 . Este grupo tiene principalmente sedimentos limo-arcillosos porque fue muestreado en la zona media del estuario, con poca influencia costera o aluvial. Al grupo 2 pertenecen los gráficos del $\mathrm{E}$ al $\mathrm{H}$ de la figura 8 y figura 9 . Estos presentan una disminución considerable en la cantidad de finos (las muestras presentan aprox. $35 \% \mathrm{de}<3,75 \varnothing$ ). Estas muestras fueron colectadas en áreas de transición entre el estuario y la costa o la planicie aluvial, lo que hace más factible materiales arenosos a gravosos.

Las curvas tienen una fuerte tendencia hacia una asimetría negativa, excepto la muestra E (Fig. 8), la cual está muy influenciada por los sedimentos arenosos que acarrea la marea. De esta manera, con sus variantes, el principal mecanismos de transporte es la suspensión, sin dejar de lado la tracción (saltación), sobre todo en A a D (Fig. 9).
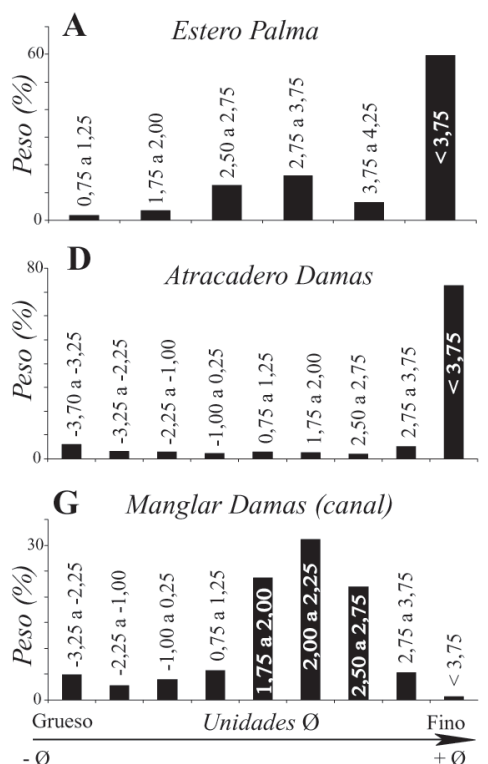
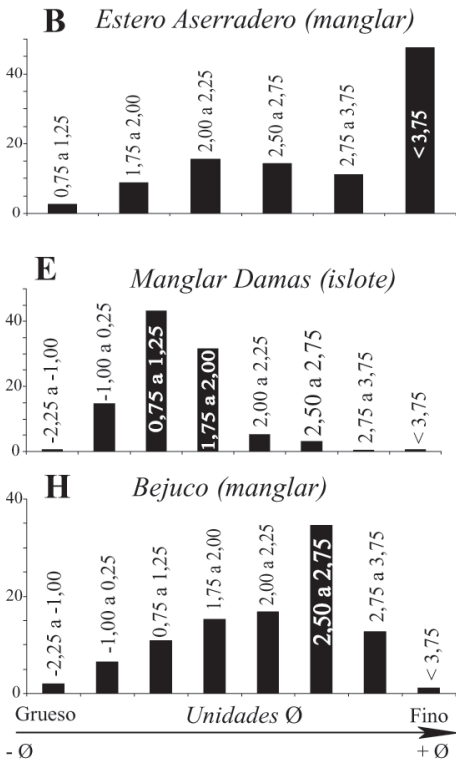
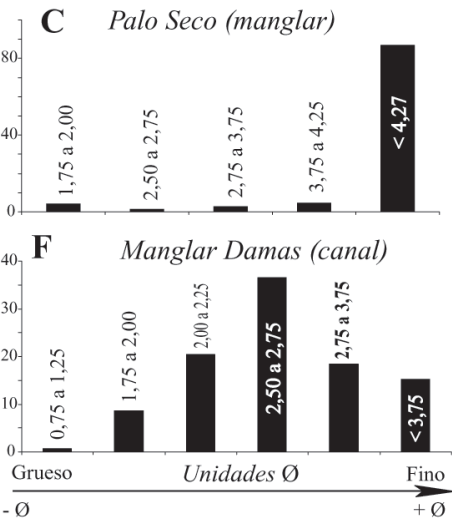

Fig. 8: Histograma de distribución granulométrica para las facies estuarinas. 


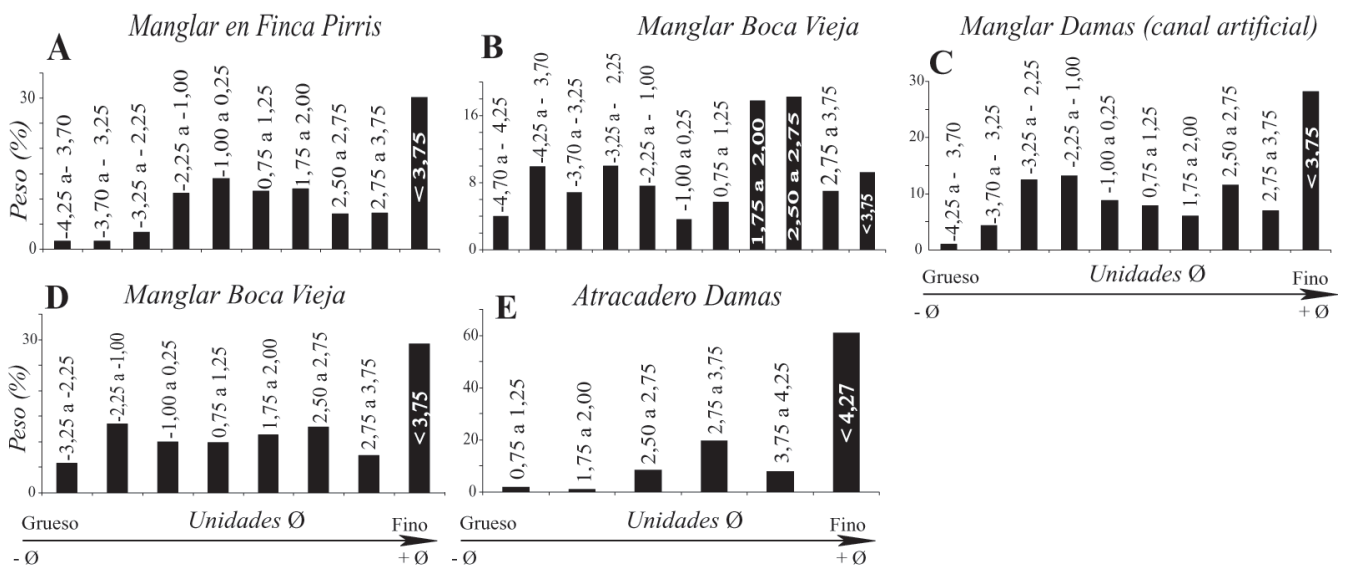

Fig. 9: Histograma de distribución granulométrica para las facies estuarinas.

Prácticamente todas las muestras son polimodales (Figs. 8 y 9). La mediana tiene valores inferiores a 1. La moda presenta dos tendencias, un grupo de muestras tienen modas que oscilan entre 0,75 a $2,75 \varnothing$ y otro grupo con modas $<3,75 \varnothing$. La selección es de regular a buena. Las curvas son en algunos casos angulosas (p.ej. A a D, Fig. 8) y en otras más bien achatadas (A a D, Fig. 9).

Los sedimentos tienen coloraciones grisáceas y presentan materia orgánica. Las estructuras sedimentarias que se observan principalmente son los restos de actividad de organismos y ondulitas.

Los sedimentos derivan de varias fuentes incluyendo la cuenca de drenaje en la zona continental adyacente, la plataforma marina (mareas y oleaje), la erosión del estuario y la actividad biológica del sistema. Sin embargo, en la zona los sedimentos estuarinos están siendo intensamente erosionados. Las mareas y el oleaje entran bastante adentro del manglar erosionando y transportando los sedimentos finos que lo constituyen. En su lugar se están depositando las arenas de las facies de playa. Evidencias de este proceso de observan en Bejuco, Esterillos y Damas. Esto se debe a un cambio en la línea de costa, la cual está migrando tierra adentro.

\section{Facies de barra arenosa (islas barrera)}

Las barras combinadas con sus ambientes sedimentarios asociados, tales como manglares, planos de marea y deltas de marea conforman un sistema de barrera, las cuales se forman en sistemas mesomareales, como es el caso de la zona de estudio.

En el área entre Esterillos y Quepos se presentan una serie de barras arenosas que son las responsables de la permanencia de los estuarios que se instalan tras estas. Las dos barras más importantes en cuanto a tamaño son la Palo Seco y la Damas.

Los sedimentos que conforman las partes subaéreas de las barras son de color beige oscuro a café claro. La distribución granulométrica de es similar al de las facies de playa, estos materiales van desde los 1,75 a $<3,75 \varnothing$, y 2,00 a $2,75 \varnothing$ como tamaños más comunes (Fig. 10). La selección es muy buena.

En forma general, las muestras colectadas en las facies de barra arenosa tienen comportamientos granulométricos similares, son curvas simétricas, excepto D (Fig. 10), con tendencias a formar campanas de Gauss en su distribución. Estas facies están formadas principalmente por arenas finas depositadas por la acción de las olas y las mareas. El sedimento es transportado principalmente por las corrientes litorales, en la zona costera se produce el transporte tanto por suspensión como por tracción.

La mediana tiene valores inferiores 0,300 , la moda se encuentra entre 2,50 y $3,75 \varnothing$. En cuanto a la angulosidad las gráficas tienden a ser achatadas. 


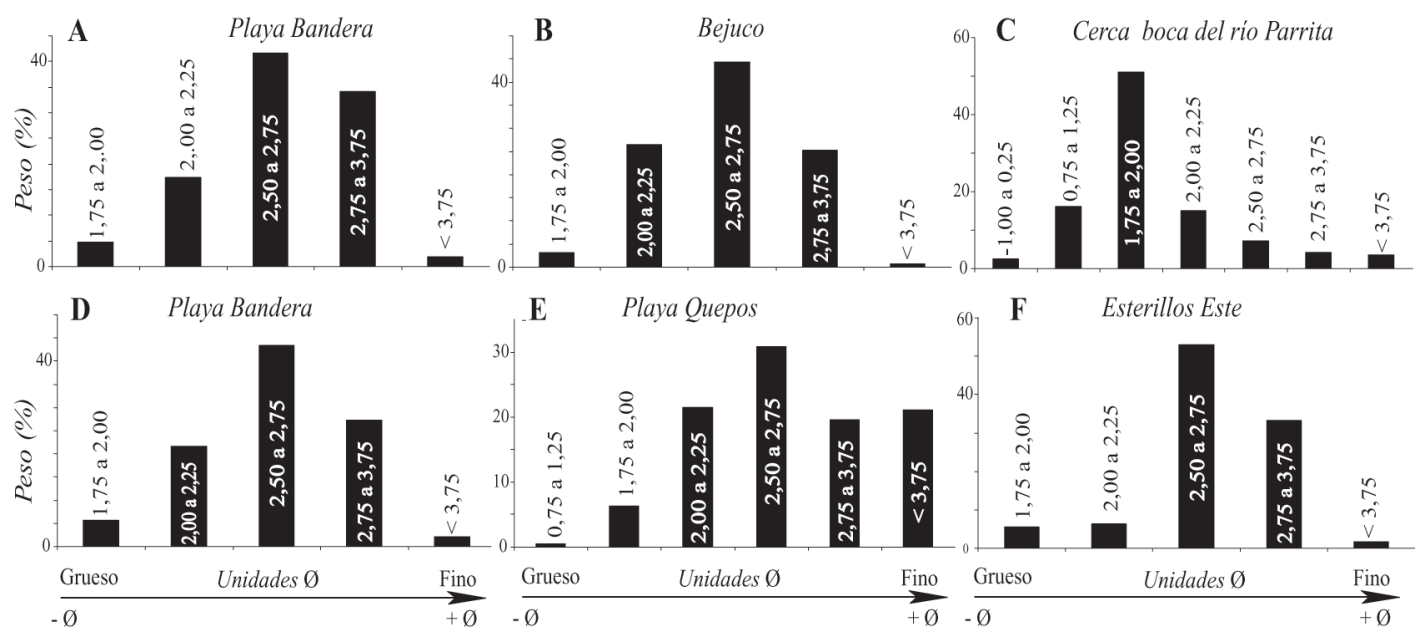

Fig. 10: Histograma de distribución granulométrica para las facies de isla barrera (barrier island).

Las principales estructuras sedimentarias que se presentan son riples, crecent marks y laminación paralela y cruzada. La berma permanente puede llegar a tener 20 a $30 \mathrm{~cm}$ de altura o estar ausente en algunos tramos. En los casos que se presenta se encuentra colonizada por vegetación. Además, en la barra Palo Seco se presentan tres secuencias de paleo-bermas con tamaños que no sobrepasan los $30 \mathrm{~cm}$ de altura.

El origen de estas barras se debe a la depositación de sedimento transportado por las corrientes marinas, el oleaje y las mareas. En el caso de la zona de estudio las barras tienen una ubicación NW-SE debido a que las fuertes corrientes litorales de la zona tienen esa misma dirección. Las barras en costas mesomareales (rango mareal $<2$ metros) se rompen por frecuentes entradas mareales (canales de marea o tidal inlets) donde deltas de marea se forman en los estuarios en el lado trasero de la barra. Un ejemplo de esto es el delta de marea que se formó luego de la ruptura de una parte de la barra Damas. La existencia de dicho delta de marea se puede inferir de los entrevistas realizadas a los hombres que navegan por las aguas del manglar de Damas-Palo-Seco. Ellos afirman que no es posible navegar en dicha zona durante la marea baja, debido a que hay mucho lodo cerca de la superficie.

\section{DISCUSIÓN}

Los procesos sedimentarios fuera de faja de meandros (también llamada planos de inundación, llanura aluvial o floodplains) están dominados por la acreción vertical derivada de un flujo de sedimento introducido por canales o desbordamiento de los canales principales (depósitos de inundación) y en algunos casos el flujo de agua escapa de los canales secundarios (crevasses splays). La planicie aluvial es una zona donde principalmente se produce despositación, también se establece un flujo en los planos aluviales que produce erosión y retrabajo de los últimos sedimentos depositados. Los procesos de depositación/erosión se desarrollan principalmente durante la estación lluviosa, época en la cual se producen fuertes eventos de inundación en la zona.

Los canales meándricos sinuosos están dominados por los procesos de acreción lateral, proceso por medio del cual se forman depositos de point-bar principalmente. Se presentan lag deposits en el fondo del cauce, los cuales están siendo continuamente erosionados /depositados.

Las facies de braided river de baja sinuosidad que se presentan en parte alta del río Parrita están dominados por acreción lateral, vertical y longitudinal, la cual conlleva la formación de 
barras laterales y longitudinales, como principales estructuras depositacionales.

Las facies de paleo-canal meándrico se depositaron cuando se produjo la migración la faja de meandros dentro de la llanura aluvial, esto debido a un proceso de migración lateral. Actualmente, estas facies están siendo modificadas y en parte erosionadas por los procesos sedimentarios naturales y por el uso del suelo en la zona.

Las facies lacustres se producen cuando los planos aluviales son inundados ya sea por el rebalse de los ríos o por el levantamiento del nivel freático.

Las facies de playa y las de barra arenosa se producen por la conjunción de las corrientes litorales (NW-SE), el oleaje y las mareas que permiten la depositación de grandes cantidades de sedimento, principalmente arenoso.

Las desembocaduras de los ríos en la zona de estudio corresponden con sistemas estuarinos, en los cuales se desarrolla un ecosistema de manglar. Pueden ser clasificados por del tipo bar-built estuary debido a que deben su existencia a la presencia de barras arenosas que dan la protección necesaria para que se desarrollen y sean colonizados.

\section{AGRADECIMIENTOS}

A Natalia Zamora por la colaboración durante parte del trabajo de campo. A Luis Obando por sus comentarios y préstamo de materiales. A los proyectos de investigación \# 113-A2-527 y 113-90-071 de la Universidad de Costa Rica por el financiamiento parcial del trabajo de campo.

\section{REFERENCIAS}

PETTIJOHN, F., J., POTTER, P., E., \& SIEVER, R., 1973: Sand and Sandstones. -618 págs. $2^{\mathrm{a}}$ ed. Springer, Berlin.

\section{APÉNDICE}

Fórmulas utilizadas para el cálculo de los parámetros granulométricos estadísticos (tomado de Pettijohn et al., 1973).

\section{TENDENCIA CENTRAL}

\section{Mediana}

$\mathrm{Md}=\mathrm{Q}_{2}$, donde $\mathrm{Q}_{2}$ el diámetro del $50 \%$

(Trask, 1930).

\section{Moda}

$\mathrm{M}=$ punto de mayor altura en la curva (unidades $\varnothing$ ).

\section{CLASIFICACIÓN Y DISPERSIÓN}

\section{Sorteo}

$\mathrm{So}=\sqrt{ } \mathrm{Q}_{1} / \mathrm{Q}_{3}$, donde $\mathrm{Q}_{1}$ el diámetro del $25 \%$ y Q3 el diámetro del 75\% (Trask, 1930).

\section{Desviación cuartilar}

$\mathrm{DQ}_{\mathrm{a}}=\mathrm{Q}_{1}-\mathrm{Q}_{3} / 2$, donde $\mathrm{Q}_{1}$ el diámetro del $25 \%$ y $\mathrm{Q}_{3}$ el diámetro del 75\% (Krumbein, 1936).

\section{ASIMETRÍA}

Asimetría aritmética

$\mathrm{Sk}_{\mathrm{a}}=\mathrm{Q}_{1}+\mathrm{Q}_{3}-2 \mathrm{Md} / 2$ (Krumbein, (1939)

Asimetría geométrica

$\mathrm{SK}_{\mathrm{g}}=\sqrt{ } \mathrm{Q}_{1} * \mathrm{Q}_{3} / \mathrm{Md}^{2}$ (Trask, 1939).

\section{ANGULOSIDAD}

\section{Angulosidad aritmética}

$\mathrm{Kq}_{\mathrm{a}}=\mathrm{Q}_{1}-\mathrm{Q}_{3} / 2\left(\mathrm{P}_{10}-\mathrm{P}_{90}\right)$, donde $\mathrm{P}_{10}$ es el diáme-

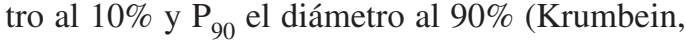
(1938). 
Cuadro 1

Terminología e intervalos de clase para

la caracterización granulométrica de sedimentos

(Modificado de Pettijohn et al., 1973)

\begin{tabular}{|c|c|c|}
\hline Milímetros & Unidades $\varnothing$ & Clase \\
\hline 4096 & -12 & \\
\hline 1024 & -10 & BLOQUES \\
\hline 256 & -8 & \\
\hline 64 & -6 & GRAVA \\
\hline 32 & $-5,0$ & GRUESA \\
\hline 16 & -4 & GRAVA \\
\hline 4 & -2 & MEDIA \\
\hline 3,36 & $-1,75$ & \\
\hline 2,83 & $-1,5$ & GRAVA \\
\hline 2,38 & $-1,25$ & FINA \\
\hline 2,00 & $-1,0$ & \\
\hline 1,68 & $-0,75$ & \\
\hline 1,41 & $-0,50$ & ARENA \\
\hline 1,19 & $-0,25$ & MUY GRUESA \\
\hline 1,00 & 0,0 & \\
\hline 0,84 & 0,25 & \\
\hline 0,71 & 0,5 & ARENA \\
\hline 0,59 & 0,75 & GRUESA \\
\hline 0,50 & 1,0 & \\
\hline 0,42 & 1,25 & \\
\hline 0,35 & 1,5 & ARENA \\
\hline 0,30 & 1,75 & MEDIA \\
\hline 0,25 & 2,0 & \\
\hline 0,210 & 2,25 & \\
\hline 0,177 & 2,5 & ARENA \\
\hline 0,149 & 2,75 & FINA \\
\hline 0,125 & 3,0 & \\
\hline 0,105 & 3,25 & \\
\hline 0,088 & 3,5 & ARENA \\
\hline 0,074 & 3,75 & MUY FINA \\
\hline 0,0625 & 4,0 & \\
\hline 0,053 & 4,25 & \\
\hline 0,044 & 4,5 & LIMO \\
\hline 0,037 & 4,75 & GRUESO \\
\hline 0,031 & 5,0 & \\
\hline 0,0156 & 6,0 & \\
\hline 0,0078 & 7,0 & LIMO MEDIO \\
\hline 0,0039 & 8,0 & A FINO \\
\hline 0,0020 & 9,0 & \\
\hline 0,00098 & 10,0 & \\
\hline 0,00049 & 11,0 & ARCILLA \\
\hline 0,00024 & 12,0 & \\
\hline 0,00012 & 13,0 & \\
\hline 0,00006 & 14,0 & \\
\hline
\end{tabular}

